\title{
Biosynthetic Process
}

National Cancer Institute

\section{Source}

National Cancer Institute. Biosynthetic Process. NCI Thesaurus. Code C17702.

Formation of a chemical compound by enzymes, either in the organism (in vivo) or by

fragments or extracts of cells (in vitro). 